\title{
On some arithmetic properties of polynomial expressions involving Stirling numbers of the second kind
}

\author{
by \\ Martin Klazar (Praha) and Florian LuCa (Morelia)
}

1. Introduction. Stirling numbers of the second kind $S(n, k)$, where $k, n \in \mathbb{N}=\{1,2, \ldots\}$, count partitions of the set $[n]=\{1, \ldots, n\}$ into $k$ nonempty disjoint sets. For example, $S(n, 1)=1$ and $S(n, 2)=2^{n-1}-1$ for every $n \in \mathbb{N}$. More generally,

$$
S(n, k)=\sum_{i=1}^{k} \frac{(-1)^{k-i}}{(k-i) ! i !} i^{n} .
$$

The proof of this well-known expansion can be found in Stanley [19, p. 34] or many other sources.

$S(n, k)$ belong to the most popular combinatorial numbers and as such are subject of many articles. A small sample is $[2,3,5,10,11,17,20,21]$. Here we are inspired by the following questions. For a fixed $k \in \mathbb{N}$, are there infinitely many squares in the sequence $(S(n, k))_{n \geq 1}$ ? What about higher powers? And what about taking instead of $S(n, k)$ a polynomial expression in it or in several Stirling numbers $S\left(n, k_{1}\right), S\left(n, k_{2}\right), \ldots, S\left(n, k_{m}\right)$ ? The only results known to us dealing with these or related diophantine problems on $S(n, k)$ were obtained by Brindza and Pintér [4] and Pintér [13, 14]. We review them briefly and then summarize our theorems. The main tools that we use are the results of Corvaja and Zannier [6] which are described in Section 2. Our results are proved in Section 3.

Pintér proved in [13] that for any fixed $a \in \mathbb{N}$, if $S(n, n-a)$ is an $m$ th power, $m \geq 3$, then $n<C$ where $C=C(a)$ is an effectively computable constant. He also proved an analogous result for the relation $S(n, n-a) \in S$ where $S$ is the set of positive integers composed only of primes from a fixed finite set. In [14] he proved that for all fixed integers $1<a<b$ the solutions of

2000 Mathematics Subject Classification: Primary 11B73; Secondary 11D61.

M. Klazar supported by the project LN00A056 of the Ministry of Education of the Czech Republic.

F. Luca supported by the grant SEP-CONACYT 37259-E. 
the equation $S(m, a)=S(n, b)$ satisfy $\max (m, n)<C b(\log b)^{3} \log (b ! / a !) \log a$ where $C$ is an effectively computable absolute constant. Brindza and Pintér [4] considered the equations $S(x, x-a)=b y^{z}$ and $S(x, a)=b y^{z}$ with parameters $a, b \in \mathbb{N}$ and unknowns $x, y, z \in \mathbb{N}$. As for the first equation, they proved that if $(x, y, z)$ is a solution with $x>2 b 16^{a} a^{8 a}$ and $y>1$, then $z(7.5+\log z)^{-2}<11000(\log b+8 a \log a+3 a)$. (The same bound is also proved for Stirling numbers of the first kind $s(n, k)$ which count the permutations of $1, \ldots, n$ with $k$ cycles.) Further, if $z \geq 3$ is fixed or $z=2$ and $a \neq 1,3$, then $\max (x, y)$ can be effectively bounded in terms of $a$ and $b$. As for the second equation, they proved that in all solutions $(x, y, z)$ with $y>1$ (also $a>1$ ), $z$ is bounded by a constant that is effectively computable in terms of $a$ and $b$. The main tool used in all these results is the theory of linear forms in logarithms.

Now we state our results and begin with two theorems on the "Stirlinglike" numbers $T(n, k)$. These are given by

$$
T(n, k)=\sum_{i=1}^{k} t(k, i) i^{n},
$$

where $t(k, i) \in \mathbb{Q}, 1 \leq i \leq k$, are some fixed nonzero constants.

Theorem 1.1. For any two fixed integers $k_{1}$ and $k_{2}, 1<k_{1}<k_{2}$, the relation

$$
\frac{T\left(n, k_{2}\right)}{T\left(n, k_{1}\right)} \in \mathbb{Z}
$$

holds for only finitely many $n \in \mathbb{N}$.

Theorem 1.2. Suppose $d>1$ is an integer, $a_{1}, \ldots, a_{m} \in \mathbb{N}$ are $m$ positive integers, not all divisible by $d$, and $1<k_{1}<\ldots<k_{m}$ are $m$ distinct integers. Then the diophantine equation

$$
T\left(n, k_{1}\right)^{a_{1}} T\left(n, k_{2}\right)^{a_{2}} \ldots T\left(n, k_{m}\right)^{a_{m}}=x^{d}
$$

has only finitely many solutions $(n, x) \in \mathbb{N}^{2}$.

Our remaining theorems deal with the more particular Stirling numbers $S(n, k)$, but in much more general expressions. In the next theorem $\psi$ is a ring isomorphism, defined in Proposition 2.4, between the power sums and the ring $\mathbb{Q}\left[X_{p}: p \in \mathcal{P}\right]$ of rational polynomials in countably many variables indexed by the primes.

Theorem 1.3. Let $P \in \mathbb{Q}\left[Y_{1}, \ldots, Y_{m}\right]$ be a nonconstant polynomial and $t \in \mathbb{N}$. There exists a constant $C=C(m, t)>0$ such that if $C<k_{1}<\ldots$ $<k_{m}$ are $m$ distinct integers, then the polynomial

$$
P\left(\psi\left(S\left(\nu, k_{1}\right)\right), \ldots, \psi\left(S\left(\nu, k_{m}\right)\right)\right)
$$

depends on at least $t$ variables. 
Corollary 1.4. Let $\theta>23 / 42$ and $P \in \mathbb{Q}\left[Y_{1}, \ldots, Y_{m}\right]$ be a nonconstant polynomial. There exists a constant $C=C(m, \theta)>0$ such that if $C<k_{1}<$ $\ldots<k_{m}$ are $m$ distinct integers, then

$$
\left|P\left(S\left(n, k_{1}\right), \ldots, S\left(n, k_{m}\right)\right)\right|>\left(k_{1}-k_{1}^{\theta}\right)^{n} \quad \text { for every } n \geq n_{0} .
$$

TheOREM 1.5. Let $P, Q \in \mathbb{Q}\left[Y_{1}, \ldots, Y_{m}\right]$ be two polynomials such that $P$ does not divide $Q$. There exists a constant $C=C(m)>0$ depending only on $m$ such that if $C<k_{1}<\ldots<k_{m}$ are $m$ distinct integers, then

$$
\frac{Q\left(S\left(n, k_{1}\right), \ldots, S\left(n, k_{m}\right)\right)}{P\left(S\left(n, k_{1}\right), \ldots, S\left(n, k_{m}\right)\right)} \in \mathbb{Z}
$$

for only finitely many $n \in \mathbb{N}$.

Theorem 1.6. Let $d \in \mathbb{N}, d>1$, and $P \in \mathbb{Q}\left[Y_{1}, \ldots, Y_{m}\right]$ be a polynomial which is not a dth power in $\mathbb{Q}\left[Y_{1}, \ldots, Y_{m}\right]$. There is a constant $C=C(m, \operatorname{deg}(P))$ depending only on $m$ and the degree of $P$ such that if $C<k_{1}<\ldots<k_{m}$ are $m$ distinct numbers, then the diophantine equation

$$
P\left(S\left(n, k_{1}\right), \ldots, S\left(n, k_{m}\right)\right)=x^{d}
$$

admits only finitely many solutions $(n, x) \in \mathbb{N}^{2}$.

TheOREM 1.7. If $P \in \mathbb{Q}\left[Y_{1}, \ldots, Y_{m}\right]$ is not a perfect power in $\mathbb{Q}\left[Y_{1}, \ldots\right.$ $\left.\ldots, Y_{m}\right]$, then there exists a constant $C_{1}=C_{1}(P)$ depending only on $P$ such that if $C_{1}<k_{1}<\ldots<k_{m}$ are $m$ distinct integers, then the diophantine equation

$$
P\left(S\left(n, k_{1}\right), \ldots, S\left(n, k_{m}\right)\right)=x^{d}
$$

has only finitely many solutions $(n, x, d) \in \mathbb{N}^{3}, x, d>1$.

Theorems 1.1 and 1.2 are straightforward applications of the results of Corvaja and Zannier [6] on power sums. The proofs of Theorems 1.3-1.7 are more technical and require besides [6] our Propositions 3.1 and 3.3. (The proof of Theorem 1.7 also uses some bounds from the theory of linear forms in logarithms.) Proposition 3.1 proves that certain polynomial systems $\left\{P_{i} \in \mathbb{C}\left[X_{1}, \ldots, X_{m}\right]: i=1, \ldots, m\right\}$ are invertible. Proposition 3.3 shows that systems $\left\{S\left(n, k_{i}\right): i=1, \ldots, m\right\}$ can be reduced, by restriction to appropriate sets of primes, to such polynomial systems. To obtain these sets we apply bounds on numbers of primes in short intervals.

Since $S(n, k)$ are quite special power sums, the independence of their coefficients $(-1)^{k-i} /(i !(k-i) !)$ given in (1) (see the application of Lemma 3.2 in the proof of Proposition 3.3 for the exact meaning of "independence") enables one to handle quite general polynomial expressions. We think that our methodology may be useful also for some power sums more general than $S(n, k)$, especially for those arising in combinatorial enumeration.

But some independence of the coefficients is necessary. Note that Theorem 1.1 cannot be generalized much more towards Theorem 1.5 because 
if $T_{0}(n, k)=k^{n}+(k-1)^{n}+\ldots+1^{n}, Q\left(X_{1}, \ldots, X_{4}\right)=X_{1}-X_{2}$, and $P\left(X_{1}, \ldots, X_{4}\right)=X_{3}-X_{4}$, then

$$
\frac{Q\left(T_{0}(2 k, n), T_{0}(2 k-1, n), T_{0}(k, n), T_{0}(k-1, n)\right)}{P\left(T_{0}(2 k, n), T_{0}(2 k-1, n), T_{0}(k, n), T_{0}(k-1, n)\right)}=2^{n} \in \mathbb{Z}
$$

for all $k, n \in \mathbb{N}$ although $P$ does not divide $Q$. Similarly, $T_{0}(n, k)$ and $P\left(X_{1}, X_{2}\right)=X_{1}-X_{2}$ show that the monomial on the left hand side of (3) cannot be in general replaced by a sum of two monomials: although $P$ is linear, $P\left(T_{0}(k, n), T_{0}(k-1, n)\right)=x^{d}$ has infinitely many solutions $(n, x) \in \mathbb{N}^{2}$ for any fixed $d>1$ and $k \in \mathbb{N}$.

Recall this notation: $\mathbb{N}=\{1,2, \ldots\}$ are positive integers, $\mathbb{Z}$ is the set of all integers, $\mathbb{Q}$ are rational numbers, $\mathbb{C}$ are complex numbers, if $m \in \mathbb{N}$ then $[m]=\{1, \ldots, m\}$, and $\mathcal{P}=\{2,3,5,7,11, \ldots\}$ is the set of prime numbers.

2. The results of Corvaja and Zannier. Our presentation of the results in [6] is somewhat more "formal" as it separates the power sums as integer sequences and their syntactic descriptions by the exponential polynomials. Let $\mathcal{E}$ be the set of all finite rational linear combinations

$$
\alpha=\alpha(\nu)=\sum_{i=1}^{k} c_{i} a_{i}^{\nu}
$$

where the $c_{i} \in \mathbb{Q}$ are all nonzero, $a_{1}>\ldots>a_{k}>0$ are distinct positive integers, $k \geq 0$ (the empty sum being 0 ), and $\nu$ is a formal variable. The integers $a_{i}$ are the roots of $\alpha$, the rationals $c_{i}$ are its coefficients, and $k$ is its rank. $\mathcal{E}$ is, with the obvious addition and multiplication, a commutative integral domain with 1 which extends the field $\mathbb{Q}$. One more operation will be of importance. The substitutions $\nu \mapsto e+d \nu$, where $e, d \in \mathbb{Z}$ and $d>0$, act on $\mathcal{E}$ by transforming $c \cdot a^{\nu}$ to $c a^{e} \cdot\left(a^{d}\right)^{\nu}$. For example,

if $\alpha(\nu)=\frac{1}{3} \cdot 4^{\nu}-3^{\nu}+\frac{7}{6} \cdot 1^{\nu} \quad$ then $\quad \alpha(2 \nu-1)=\frac{1}{12} \cdot 16^{\nu}-\frac{1}{3} \cdot 9^{\nu}+\frac{7}{6} \cdot 1^{\nu}$.

One turns $\alpha$ into a function $\alpha: \mathbb{N} \rightarrow \mathbb{Q}$ by substituting positive integers $n \in \mathbb{N}$ for $\nu$. It is clear that two different $\alpha_{1}, \alpha_{2} \in \mathcal{E}$ produce two different functions; one even has $\alpha_{1}(n)>\alpha_{2}(n)$ or vice versa for all $n>n_{0}$. Thus one can view $\mathcal{E}$ in two ways, as a ring of exponential polynomials or as a ring of particular functions from $\mathbb{N}$ to $\mathbb{Q}$.

The following result is Theorem 1 of [6].

Proposition 2.1. If $\alpha, \beta \in \mathcal{E}$ are such that $\alpha(n) / \beta(n) \in \mathbb{Z}$ for infinitely many $n \in \mathbb{N}$, then there is a $\gamma \in \mathcal{E}$ such that

$$
\alpha=\beta \cdot \gamma .
$$

The following result is Corollary 1 of Theorem 2 in [6]. 
Proposition 2.2. If $\alpha \in \mathcal{E}, d>1$ is an integer, and $\alpha(n)=x^{d}$ has infinitely many solutions $(n, x) \in \mathbb{N}^{2}$, then there is an integer $e \in$ $\{0,1, \ldots, d-1\}$ and an element $\beta \in \mathcal{E}$ such that

$$
\alpha(e+d \nu)=\beta(\nu)^{d} .
$$

The following result is a part of Corollary 2 of Theorem 3 in [6]; in [6] a stronger approximation result is given.

Proposition 2.3. Suppose that $\alpha \in \mathcal{E}$ has rank at least 2 and its two leading roots $a_{1}>a_{2}$ are coprime. Then, for every fixed integer $d>1$, the equation $\alpha(n)=x^{d}$ has only finitely many solutions $(n, x) \in \mathbb{N}^{2}$.

This is a consequence of Proposition 2.2. Indeed, suppose that there are infinitely many solutions. Then (5) holds. So $\beta(\nu)$ has rank at least 2 as well. If $b_{1}>b_{2}$ are the two leading roots of $\beta(\nu)$, then $\beta(\nu)^{d}$ has two leading roots $b_{1}^{d}>b_{1}^{d-1} b_{2}$ with the gcd at least $b_{1}^{d-1} \geq b_{1} \geq 2$. On the other hand, the two leading roots of $\alpha(e+d \nu)$ are $a_{1}^{d}>a_{2}^{d}$ and are coprime. Hence (5) cannot hold, which is a contradiction.

The simple criterion of Proposition 2.3 answers the question about perfect powers in $(S(n, k))_{n \geq 1}$. By (1), for $k \geq 2$ the two leading roots of $S(\nu, k)$ are the coprime numbers $k>k-1$. So, $S(n, k)=x^{d}$ has for fixed $k, d \geq 2$ only finitely many solutions $(n, x) \in \mathbb{N}^{2}$. Combining this with the result of Brindza and Pintér [4] (see the previous section) we find that for every fixed $k \geq 2$ the equation $S(n, k)=x^{d}$ has only finitely many solutions $(n, x, d) \in \mathbb{N}^{3}$ with $x, d>1$.

There is yet a third way of looking at the $\operatorname{ring} \mathcal{E}$ which we state in the form of the next proposition; its proof is trivial.

Proposition 2.4. Let the mapping $\psi: \mathcal{E} \rightarrow \mathbb{Q}\left[X_{p}: p \in \mathcal{P}\right]$ be defined by $\psi\left(c 1^{\nu}\right)=c$ for $c \in \mathbb{Q}, \psi\left(p^{\nu}\right)=X_{p}$ for $p \in \mathcal{P}$, and by the multiplicative and additive extension on the remaining elements of $\mathcal{E}$. Then $\psi$ is a ring isomorphism between $\mathcal{E}$ and $\mathbb{Q}\left[X_{p}: p \in \mathcal{P}\right]$.

For example,

$$
\psi\left(-\frac{7}{90} \cdot 24^{\nu}+10^{\nu}+5 \cdot 9^{\nu}-10 \cdot 1^{\nu}\right)=-\frac{7}{90} X_{2}^{3} X_{3}+X_{2} X_{5}+5 X_{3}^{2}-10 .
$$

The isomorphism $\psi$ is a standard tool for dealing with exponential polynomials and goes back at least to Ritt [16]. See van der Poorten [15, 3.2] for more information.

We need to show that certain relations of type (4) or (5) are impossible. We accomplish this by interpreting them via $\psi$ as polynomial identities. See Luca and Walsh [12] for similar applications.

3. The proofs. In the proofs of Theorems 1.1 and 1.2 we need the well-known Bertrand's postulate. It asserts that for every $n \in \mathbb{N}, n \geq 2$, the 
interval $(n / 2, n]$ contains at least one prime number. A simple proof was given by Erdős [7]. A nice presentation of this proof is in Aigner and Ziegler $[1$, pp. $7-12]$.

Proof of Theorem 1.1. Suppose, for a contradiction, that (2) holds for infinitely many $n \in \mathbb{N}$. By Proposition 2.1, we have in $\mathcal{E}$ the identity

$$
T\left(\nu, k_{2}\right)=T\left(\nu, k_{1}\right) \cdot \gamma
$$

with a nonzero $\gamma \in \mathcal{E}$. Hence $k_{2}=a k_{1}$ where $a \in \mathbb{N}$ is the leading root of $\gamma$. Since $k_{2}>k_{1}$, we have $k_{2} \geq 2 k_{1}$. By Bertrand's postulate, there is a prime number $p$ such that $k_{2} \geq p>k_{1}$. From (6),

$$
A=B \cdot C
$$

where $A=\psi\left(T\left(\nu, k_{2}\right)\right), B=\psi\left(T\left(\nu, k_{1}\right)\right)$, and $C=\psi(\gamma)$ are polynomials from $\mathbb{Q}\left[X_{p}: p \in \mathcal{P}\right]$. The variable $X_{p}$ does not appear in $B$ but it appears exactly once in $A$, in the monomial $t\left(k_{2}, p\right) X_{p}$ (remember that all $t(k, i)$ are nonzero). Since $k_{1}>1, B$ is a nonconstant polynomial. If $X_{p}$ does not appear in $C$, the identity is patently impossible. If $X_{p}$ appears in $C$, it is also impossible because then $B \cdot C$ has at least one monomial of the type $D X_{p}$, where $D$ is a nonconstant monomial in the variables distinct from $X_{p}$, but $A$ contains no such monomial. We have a contradiction.

Proof of Theorem 1.2. Without loss of generality we can assume that no exponent $a_{i}$ in (3) is divisible by $d$. We fix an $m$-tuple $1<k_{1}<\ldots<k_{m}$ of distinct integers and an $m$-tuple of positive integers $a_{1}, \ldots, a_{m}$ none of which is divisible by $d>1$, and assume that

$$
T\left(n, k_{1}\right)^{a_{1}} \ldots T\left(n, k_{m}\right)^{a_{m}}=x^{d}
$$

has infinitely many solutions $(n, x) \in \mathbb{N}^{2}$. We show that this leads to a contradiction.

By Proposition 2.2,

$$
T\left(e+d \nu, k_{1}\right)^{a_{1}} \ldots T\left(e+d \nu, k_{m}\right)^{a_{m}}=\gamma^{d}
$$

for some $e \in\{0,1, \ldots, d-1\}$ and $\gamma \in \mathcal{E}$. Applying $\psi$, we obtain the identity

$$
\prod_{j=1}^{m}\left(\sum_{i=1}^{k_{j}} t\left(k_{j}, i\right) i^{e} X(i)^{d}\right)^{a_{j}}=Q^{d}
$$

where $X(i)=\prod_{p \mid i} X_{p}^{m_{p}(i)}\left(m_{p}(i)\right.$ is the exponent of $p$ in the prime decomposition of $i$ ) and $Q=\psi(\gamma) \in \mathbb{Q}\left[X_{p}: p \in \mathcal{P}\right]$. Let $q$ be the maximum prime number in the interval $\left(k_{m} / 2, k_{m}\right]$. By Bertrand's postulate, $q$ exists. The variable $X_{q}$ appears in each of the $m$ factors on the left hand side of (7) at most once, possibly in the summand $t\left(k_{j}, q\right) q^{e} X(q)^{d}=t\left(k_{j}, q\right) q^{e} X_{q}^{d}$, 
and it does appear in the last $m$ th factor. Distinguishing $X_{q}$ and denoting $R=\mathbb{Q}\left[X_{p}: p \in \mathcal{P} \& p \neq q\right]$, we write (7) in the form

$$
\prod_{j=1}^{m}\left(t\left(k_{j}, q\right) q^{e} X_{q}^{d}+B_{j}\right)^{a_{j}}=Q^{d} .
$$

Here $t\left(k_{j}, q\right)=0$ if $q>k_{j}$ and $t\left(k_{m}, q\right) \neq 0, B_{j} \in R$, and $Q \in R\left[X_{q}\right]$. Clearly, every $B_{j}$ is nonzero and except for the case $k_{j_{0}}=q$ and $k_{j_{0}-1}=q-1$ when $B_{j_{0}}=B_{j_{0}-1}$, we see that if $j_{1}>j_{2}$ then $B_{j_{1}}$ has a monomial not contained in $B_{j_{2}}$ (since all $t(k, i)$ are nonzero). Hence each two of the $B_{j}$ s with $k_{j} \geq q$ differ by more than a constant multiple. So we write (8) as

$$
B \prod_{j=1}^{s}\left(X_{q}^{d}+C_{j}\right)^{b_{j}}=Q^{d}
$$

where $B, C_{j} \in R$ are nonzero, $s \geq 1, b_{j}$ are positive integers not divisible by $d$, and $C_{j}$ are mutually distinct. This identity is impossible. The left hand side, taken as a polynomial in $X_{q}$, has $d s \geq 2$ distinct roots none of which has multiplicity divisible by $d$. But each root of the right hand side (if any) has multiplicity divisible by $d$. We have obtained a contradiction.

A polynomial $P \in \mathbb{C}\left[X_{1}, \ldots, X_{m}\right]$ depends on $X_{i}$ if $\partial P / \partial X_{i} \neq 0$. An interval partition $\mathcal{M}$ of $[m]$ is a partition $[m]=I_{1} \cup \ldots \cup I_{s}$ into disjoint nonempty intervals $I_{1}<\ldots<I_{s}$. A polynomial system $\left\{P_{i} \in\right.$ $\left.\mathbb{C}\left[X_{1}, \ldots, X_{m}\right]: i=1, \ldots, m\right\}$ is $\mathcal{M}$-regular if (i) for every $k$ and $i \in I_{k}$ one has $P_{i} \in \mathbb{C}\left[X_{j}: j \in I_{1} \cup \ldots \cup I_{k}\right]$, (ii) for every $k$ and $i, j \in I_{k}$ the variable $X_{j}$ appears in $P_{i}$ only in the monomial $b_{i, j} X_{j}$ ( $b_{i, j} \in \mathbb{C}$ is possibly zero), and (iii) the Jacobian $\operatorname{det}\left(\partial P_{i} / \partial X_{j}\right)$ is a nonzero constant. We call the linear tail $\sum_{j \in I_{k}} b_{i, j} X_{j}$ of $P_{i}, i \in I_{k}$, the linear part of $P_{i}$.

Suppose $\left\{P_{i}: i=1, \ldots, m\right\}$ is $\mathcal{M}$-regular. Then, by (i) and (ii), the Jacobi matrix $\left(\partial P_{i} / \partial X_{j}\right)$ has a block lower triangular form with $s$ blocks on the main diagonal (and zeros above the blocks) where the $k$ th block is a square $\left|I_{k}\right| \times\left|I_{k}\right|$ matrix $A_{k}$ of coefficients of the linear parts of the $P_{i} \mathrm{~s}$, $i \in I_{k}$. Condition (iii) is equivalent to the fact that every $A_{k}$ is regular because the Jacobian equals $\operatorname{det}\left(A_{1}\right) \ldots \operatorname{det}\left(A_{s}\right)$.

If the polynomials and their variables are indexed instead of $[m]$ by two arbitrary $m$-sets of integers $M$ and $N$, we transfer the interval partition $\mathcal{M}$ of $[m]$ to $M$ and $N$ in the obvious way and work with $\mathcal{M}$-regular systems also in this more general situation.

Proposition 3.1. Let $P \in \mathbb{C}\left[X_{1}, \ldots, X_{m}\right]$ be a nonconstant polynomial, $\mathcal{M}$ an interval partition of $[m]$ with parts $I_{1}<\ldots<I_{s}$, and $\left\{P_{i} \in\right.$ $\left.\mathbb{C}\left[X_{1}, \ldots, X_{m}\right]: i=1, \ldots, m\right\}$ an $\mathcal{M}$-regular system of polynomials. Then the following hold. 
(a) There exist $m$ polynomials $Q_{1}, \ldots, Q_{m} \in \mathbb{C}\left[X_{1}, \ldots, X_{m}\right]$ inverting the system $\left\{P_{i}: i=1, \ldots, m\right\}$, that is, $P_{i}\left(Q_{1}, \ldots, Q_{m}\right)=X_{i}$ for every $i=1, \ldots, m$.

(b) The system $\left\{Q_{i}: i=1, \ldots, m\right\}$ of (a) is $\mathcal{M}$-regular.

(c) The polynomial $P\left(P_{1}, \ldots, P_{m}\right) \in \mathbb{C}\left[X_{1}, \ldots, X_{m}\right]$ is nonconstant.

(d) If $P$ depends on some variable $X_{j}, j \in I_{s}$, then $P\left(P_{1}, \ldots, P_{m}\right)$ also depends on some variable $X_{k}, k \in I_{s}$.

Proof. Let $P, \mathcal{M}$, and $\left\{P_{i}: i=1, \ldots, m\right\}$ be as stated. For every $k=$ $1, \ldots, s$ consider the linear system

$$
\left\{\sum_{j \in I_{k}} b_{i, j} X_{j}=Y_{i}-R_{i}: i \in I_{k}\right\}
$$

in the unknowns $X_{j}, j \in I_{k}$, where the left hand sides are the linear parts of the $P_{i} \mathrm{~s}, i \in I_{k}, Y_{i}$ are new variables, and $R_{i}=P_{i}-\sum_{j \in I_{k}} b_{i, j} X_{j} \in \mathbb{C}\left[X_{j}\right.$ : $\left.j \in I_{1} \cup \ldots \cup I_{k-1}\right]$. The unique solution obtained by multiplying by $A_{k}^{-1}$ is the polynomials $X_{j}=T_{j}\left(Y_{j}: j \in I_{k} ; X_{j}: j \in I_{1} \cup \ldots \cup I_{k-1}\right), j \in I_{k}$. The first $\left|I_{1}\right|$ polynomials $T_{j}$ are linear and free of the $X$-variables. Substituting $T_{j}$ for $X_{j}, j \in I_{1}$, in $T_{j}$ with $j \in I_{2}$, we eliminate the $X$-variables in the next $\left|I_{2}\right|$ polynomials $T_{j}$. Continuing this way we eliminate from every $T_{j}$ every $X$-variable and obtain some polynomials $Q_{j}, j=1, \ldots, m$, only in the $Y$-variables. It follows by elementary properties of matrix multiplication that $\left\{Q_{j}: j=1, \ldots, m\right\}$ indeed inverts $\left\{P_{i}: i=1, \ldots, m\right\}$.

The solution process in (a) gives the system $\left\{Q_{i}: i=1, \ldots, m\right\}$ the form prescribed in the conditions (i) and (ii) of regularity. The diagonal blocks of its Jacobi matrix are just the inverse matrices $A_{k}^{-1}$. Thus its Jacobian is nonzero and equals the reciprocal of the Jacobian of $\left\{P_{i}: i=1, \ldots, m\right\}$. This proves (b).

(c) follows immediately from (a): If $P\left(P_{1}, \ldots, P_{m}\right)$ were constant, then $P\left(P_{1}\left(Q_{1}, \ldots, Q_{m}\right), \ldots, P_{m}\left(Q_{1}, \ldots, Q_{m}\right)\right)=P\left(X_{1}, \ldots, X_{m}\right)$ would be constant as well.

As for (d), we show that for a specialization of the first $\left|I_{1}\right|+\ldots+\left|I_{s-1}\right|$ variables $P\left(P_{1}, \ldots, P_{m}\right)$ becomes a nonconstant polynomial. We write $P$ as a finite sum

$$
P=\sum_{M} T_{M} \cdot M
$$

where $M \in \mathbb{C}\left[X_{j}: j \in I_{s}\right]$ are distinct monic monomials and $T_{M} \in \mathbb{C}\left[X_{j}\right.$ : $\left.j \in I_{1} \cup \ldots \cup I_{s-1}\right]$. Since $P$ depends on one of the last $\left|I_{s}\right|$ variables, there is a nonconstant $M_{0}$ with a nonzero $T_{M_{0}}$. By (c), $T_{M_{0}}^{*}=T_{M_{0}}\left(X_{j}:=P_{j}\right.$ : $j \in I_{1} \cup \ldots \cup I_{s-1}$ ) is a nonzero (but possibly constant) polynomial. We set $X_{j}:=\alpha_{j} \in \mathbb{C}, j \in I_{1} \cup \ldots \cup I_{s-1}$, so that $T_{M_{0}}^{*}$ becomes a nonzero constant. Then $P\left(X_{j}:=P_{j}: j \in I_{1} \cup \ldots \cup I_{s-1} ; X_{j}: j \in I_{s}\right)$ becomes a nonconstant 
polynomial $P^{*} \in \mathbb{C}\left[X_{j}: j \in I_{s}\right]$ and $\left\{P_{j}: j \in I_{s}\right\}$ becomes an $\mathcal{N}$-regular system $\left\{P_{j}^{*} \in \mathbb{C}\left[X_{j}: j \in I_{s}\right]: j \in I_{s}\right\}$ where $\mathcal{N}$ consists of just one part $I_{s}$. Again by $(\mathrm{c}), P\left(P_{1}, \ldots, P_{m}\right)\left(X_{j}:=\alpha_{j}: j \in I_{1} \cup \ldots \cup I_{s-1}\right)=P^{*}\left(X_{j}:=P_{j}^{*}\right.$ : $\left.j \in I_{s}\right)$ is a nonconstant polynomial.

Claim (a) of the previous proposition is a particular (and rather trivial) case of the famous Jacobian conjecture which is still open; see, for example, [8].

LEMma 3.2. Let $1 \leq k_{1}<\ldots<k_{m}$ be $m$ distinct integers and

$$
D_{m}=D_{m}\left(X_{1}, \ldots, X_{m}\right)=\operatorname{det}\left(\prod_{k=1}^{k_{m}-k_{i}}\left(k_{i}+k-X_{j}\right)\right)_{i, j=1}^{m}
$$

where for $i=m$ the product is defined as 1 . Then for every $t \in \mathbb{N}$ and $A \subset \mathbb{C}$ with $|A| \geq m\left(k_{m}-k_{1}+t\right)$ there exist $t$ mutually disjoint $m$-tuples $\left(\alpha_{i, 1}, \ldots, \alpha_{i, m}\right) \in A^{m}$ of elements of $A$, the elements in each $m$-tuple being mutually distinct, such that $D_{m}\left(\alpha_{i, 1}, \ldots, \alpha_{i, m}\right) \neq 0$ for every $i=1, \ldots, t$.

Proof. $D_{m}$ is a polynomial which has degree at most $d=k_{m}-k_{1}$ in every variable $X_{i}$. We show that $D_{m}$ is not identically zero by proving by induction on $m$ that actually $\operatorname{deg}_{X_{1}}\left(D_{m}\right)=d$ (by symmetry, this holds for every variable). For $m=1$ it is true because $D_{1}=1$. For $m>1$ we expand $D_{m}$ by the first row:

$$
D_{m}=\prod_{k=1}^{k_{m}-k_{1}}\left(k_{1}+k-X_{1}\right) \cdot E+\sum_{j=2}^{m}(-1)^{j+1} a_{1, j} M_{1, j}
$$

where $E=D_{m-1}\left(X_{2}, \ldots, X_{m}\right)$ corresponds to the $(m-1)$-tuple $k_{2}, \ldots, k_{m}$, $a_{i, j}$ is the entry of the matrix defining $D_{m}$, and $M_{i, j}$ is the minor of $a_{i, j}$. The first term is of degree $d$ in $X_{1}$ (by induction, $E$ is a nonzero polynomial), $a_{1, j}$ are $X_{1}$-free, and $\operatorname{deg}_{X_{1}}\left(M_{1, j}\right) \leq k_{m}-k_{2}<d$. Hence $\operatorname{deg}_{X_{1}}\left(D_{m}\right)=d$.

Now it suffices to prove that for every $A \subset \mathbb{C}$ with $|A| \geq m(d+1)$ we can select $m$ distinct elements $\alpha_{1}, \ldots, \alpha_{m} \in A$ such that $D_{m}\left(\alpha_{1}, \ldots, \alpha_{m}\right) \neq 0$. Since $D_{m}$ is a nonzero polynomial in $m$ variables and of degree at most $d$ in each, this follows by an easy induction on $m$ using the basic fact that every nonzero $P \in \mathbb{C}[X], \operatorname{deg}(P) \leq d$, has at most $d$ distinct roots.

For the proofs of Theorems 1.3-1.7 we need a bound on the number of primes in the interval $(x-\Delta, x)$ stronger than Bertrand's postulate. We use the fact that there exists a real number $\theta, 0<\theta<1$, and constants $\kappa, x_{0}>0$ such that

$$
\pi(x)-\pi(x-\Delta)>\kappa \Delta / \log x
$$

whenever $x>x_{0}$ and $x^{\theta}<\Delta<x$. By the result of Iwaniec and Pintz [9], this is true for every $\theta>23 / 42$. We can certainly fix $\theta$ to be $2 / 3$. 
For the next key result recall the definition of $\psi$ given in Proposition 2.4. The notation like $X_{p}:=\alpha_{p}: p \notin \mathcal{P}_{k}$ means that the variables $X_{p}$ with $p \in \mathcal{P}_{k}$ are left unevaluated and for the remaining $X_{p}$ we substitute the constants $\alpha_{p}$.

Proposition 3.3. For every $m, t \in \mathbb{N}$ there is a constant $C=C(m, t)$ $>0$ such that the following holds. For any $m$ distinct positive integers $C<$ $k_{1}<\ldots<k_{m}$ there is an interval partition $\mathcal{M}$ of $[m]$ and $t$ mutually disjoint $m$-sets of prime numbers $\mathcal{P}_{1}, \ldots, \mathcal{P}_{t}$ such that for every $k=1, \ldots, t$ and every specialization $\left\{\alpha_{p} \in \mathbb{C}: p \in \mathcal{P}\right\}$ the system of $m$ polynomials from $\mathbb{Q}\left[X_{p}: p \in \mathcal{P}_{k}\right]$

$$
\left\{\psi\left(S\left(\nu, k_{i}\right)\right)\left(X_{p}:=\alpha_{p}: p \notin \mathcal{P}_{k}\right): i=1, \ldots, m\right\}
$$

is $\mathcal{M}$-regular.

Proof. First we fix an arbitrary decreasing sequence $\theta_{1}>\theta_{2}>\ldots$ of numbers in the interval $(2 / 3,1)$. To be specific, we set

$$
\theta_{i}=\frac{5}{3}-\frac{i+2}{i+3}
$$

So $\left(\theta_{i}\right)_{i \geq 1}=\left(\frac{11}{12}, \frac{13}{15}, \frac{5}{6}, \ldots\right)$. Let $m, t \in \mathbb{N}$ be given. We fix a sufficiently large constant $C=C(m, t)>0$ meeting the following conditions, in which $x_{0}$ and $\kappa$ are the constants of bound (9) corresponding to $\theta=2 / 3$ :

$$
\begin{aligned}
C & >x_{0}, \\
k>C & \Rightarrow(m+1) k^{\theta_{1}}<\frac{1}{2} k, \\
k>C & \Rightarrow \frac{\kappa k^{\theta_{i}}}{\log k}>m\left(m(2 k)^{\theta_{i+1}}+t\right) \quad \text { for every } i=1, \ldots, m .
\end{aligned}
$$

Let $C<k_{1}<\ldots<k_{m}$ be $m$ fixed integers. We take the set $A, 1 \in A \subset$ $[m]$, defined by

$$
A=\{1\} \cup\left\{i \in[m]: i \geq 2 \& k_{i}-k_{i}^{\theta_{i}} \geq k_{i-1}\right\} .
$$

$A$ has $s \geq 1$ elements $1=a_{1}<\ldots<a_{s} \leq m$. We define the interval partition $\mathcal{M}$ of $[m]$ by

$$
\mathcal{M}=\left\{I_{1}, \ldots, I_{s}\right\}=\left\{\left[1, a_{2}-1\right],\left[a_{2}, a_{3}-1\right], \ldots,\left[a_{s-1}, a_{s}-1\right],\left[a_{s}, m\right]\right\}
$$

and set $a_{s+1}=m+1$. For $j=1, \ldots, s$ we define the interval

$$
J_{j}=\left(k_{a_{j}}-k_{a_{j}}^{\theta_{a_{j}}}, k_{a_{j}}\right] \text {. }
$$

Clearly,

$$
k_{a_{j}-1}<J_{j} \leq k_{a_{j}} .
$$

Let $K=k_{a_{j+1}-1}=\max \left\{k_{i}: i \in I_{j}\right\}$. By the definition of $A$, if $a_{j+1}-2 \in I_{j}$ then

$$
k_{a_{j+1}-2}>K-K^{\theta_{a_{j+1}-1}} \geq K-K^{\theta_{a_{j}}} .
$$


From this, if $a_{j+1}-3 \in I_{j}$ then

$$
k_{a_{j+1}-3}>k_{a_{j+1}-2}-k_{a_{j+1}-2}^{\theta_{a_{j+1}-2}} \geq K-2 K^{\theta_{a_{j}}}
$$

and so on. In the end,

$$
k_{a_{j}} \geq K-\left(\left|I_{j}\right|-1\right) K^{\theta_{a_{j}}}>K-m K^{\theta_{a_{j}}} .
$$

From this and by (11),

$$
K=k_{a_{j+1}-1}<2 k_{a_{j}}
$$

and

$$
k_{a_{j}}-k_{a_{j}}^{\theta_{a_{j}}}>K-(m+1) K^{\theta_{a_{j}}}>\frac{1}{2} K=\frac{1}{2} k_{a_{j+1}-1} .
$$

We conclude that

$$
J_{j} \subset\left(\frac{1}{2} k_{i}, k_{i}\right] \quad \text { for every } i \in I_{j} .
$$

Let $j \in \mathbb{N}, 1 \leq j \leq s$, be again arbitrary and fixed. Let $\mathcal{Q} \subset J_{j}$ be an $\left|I_{j}\right|=\left(a_{j+1}-a_{j}\right)$-element set of primes. By (15), for every $i \in I_{j}$ and $p \in \mathcal{Q}$ the variable $X_{p}$ appears in $\psi\left(S\left(\nu, k_{i}\right)\right)$ only in the linear term $\alpha_{i, p} X_{p}$. By (1), the matrix of coefficients $A_{j}$ is

$$
A_{j}=\left(\alpha_{i, p}\right)_{i \in I_{j}, p \in \mathcal{Q}}=\left(\frac{(-1)^{k_{i}-p}}{\left(k_{i}-p\right) ! p !}\right)_{i \in I_{j}, p \in \mathcal{Q}} .
$$

Let again $K=k_{a_{j+1}-1}$. The determinant $\operatorname{det} A_{j}$ is nonzero if and only if $\operatorname{det} B_{j}$ is nonzero where the matrix $B_{j}$ arises by multiplying every column $p$ of $A_{j}$ by $(-1)^{p}(K-p) ! p$ ! and every row $k_{i}$ by $(-1)^{k_{i}}$. We have

$$
B_{j}=\left(\prod_{k=1}^{K-k_{i}}\left(k_{i}+k-p\right)\right)_{i \in I_{j}, p \in \mathcal{Q}} .
$$

Hence $\operatorname{det} A_{j} \neq 0$ if and only if the polynomial $D_{\left|I_{j}\right|}$ of Lemma 3.2, corresponding to the parameters $\left\{k_{i}: i \in I_{j}\right\}$, does not vanish on the ordered $\left|I_{j}\right|$-tuple of the elements of $\mathcal{Q} . D_{\left|I_{j}\right|}$ has degree $K-k_{a_{j}}$ in every variable. By the definition of $A$ and (14),

$$
\begin{aligned}
K-k_{a_{j}} & =\sum_{r=1}^{a_{j+1}-a_{j}-1} k_{a_{j}+r}-k_{a_{j}+r-1}<\sum_{r=1}^{a_{j+1}-a_{j}-1} k_{a_{j}+r}^{\theta_{a_{j}+r}} \\
& \leq m K^{\theta_{a_{j}+1}}<m\left(2 k_{a_{j}}\right)^{\theta_{a_{j}+1}} .
\end{aligned}
$$

By (9) and (10),

$$
\left|J_{j} \cap \mathcal{P}\right|>\frac{\kappa k_{a_{j}}^{\theta_{a_{j}}}}{\log \left(k_{a_{j}}\right)} .
$$

By (12), (16), and (17),

$$
\left|J_{j} \cap \mathcal{P}\right|>m\left(K-k_{a_{j}}+t\right) \geq\left|I_{j}\right|\left(K-k_{a_{j}}+t\right) .
$$


Using Lemma 3.2, we select $t$ mutually disjoint $\left|I_{j}\right|$-sets of primes $\mathcal{Q}_{1, j} \subset$ $J_{j}, \ldots, \mathcal{Q}_{t, j} \subset J_{j}$ such that for every $\mathcal{Q}_{i, j}, 1 \leq i \leq t$, the corresponding matrix of coefficients $A_{j}$ is regular. We define, for $i=1, \ldots, t$, the desired $m$-element sets of primes as

$$
\mathcal{P}_{i}=\bigcup_{j=1}^{s} \mathcal{Q}_{i, j} .
$$

By the selection of the $\mathcal{Q}_{i, j} \mathrm{~s}$ and by (13), these sets are disjoint. Let $\left\{\alpha_{p} \in \mathbb{C}: p \in \mathcal{P}\right\}$ be any fixed specialization. By (13) and (15), for every $k=1, \ldots, t$ the Jacobi matrix of $\left\{\psi\left(S\left(\nu, k_{i}\right)\right)\left(X_{p}:=\alpha_{p}: p \notin \mathcal{P}_{k}\right): i=\right.$ $1, \ldots, m\}$ satisfies conditions (i) and (ii) of $\mathcal{M}$-regularity. Its determinant is a nonzero constant because of the selection of the $\mathcal{Q}_{i, j} \mathrm{~s}$. The proposition is proved.

Note that we have proved more than is stated (and we will need this): By (15), if $p \in \mathcal{P}_{k}$ belongs to the last part of $\mathcal{M}$, that is, $p \in \mathcal{Q}_{k, s}$, then $X_{p}$ appears in every $\psi\left(S\left(\nu, k_{i}\right)\right.$ ) (in all variables $\left\{X_{p}: p \in \mathcal{P}\right\}$ ) only in the linear term $b X_{p}$.

Proof of Theorem 1.3. It follows immediately by combining (c) of Proposition 3.1 and Proposition 3.3.

Proof of Corollary 1.4. We can use in the proof of Proposition 3.3 a decreasing sequence $\left(\theta_{i}\right)_{i \geq 1}$ in the interval $(23 / 42,1)$ such that $\theta_{1}=\theta$. Then we set $C$ to be $C(m, 1)$ of Theorem 1.3. By Theorem 1.3 and by the proof of Proposition 3.3, the polynomial

$$
Q=P\left(\psi\left(S\left(\nu, k_{1}\right)\right), \ldots, \psi\left(S\left(\nu, k_{m}\right)\right)\right)
$$

has a monomial (with nonzero coefficient) containing a power $X_{p}^{s}$ such that $s \geq 1$ and $p>k_{1}-k_{1}^{\theta}$. Thus the leading root $a \in \mathbb{N}$ of $\psi^{-1}(Q)=c a^{\nu}+\ldots$, $c \in \mathbb{Q}$ is nonzero, satisfies $a \geq p>k_{1}-k_{1}^{\theta}$ and the corollary follows.

Proof of Theorem 1.5. We set the constant $C$ to be $C(m, 1)$ of Proposition 3.3. We prove the contraposition of the implication. Suppose that $C<k_{1}<\ldots<k_{m}$ are fixed and $\frac{Q\left(S\left(n, k_{1}\right), \ldots, S\left(n, k_{m}\right)\right)}{P\left(S\left(n, k_{1}\right), \ldots, S\left(n, k_{m}\right)\right)} \in \mathbb{Z}$ for infinitely many $n \in \mathbb{N}$. By Proposition 2.1, we have in $\mathbb{Q}\left[X_{p}: p \in \mathcal{P}\right]$ the identity

$$
\psi\left(Q\left(S\left(\nu, k_{1}\right), \ldots, S\left(\nu, k_{m}\right)\right)\right)=\psi\left(P\left(S\left(\nu, k_{1}\right), \ldots, S\left(\nu, k_{m}\right)\right)\right) \cdot T
$$

for some polynomial $T$. Let $\mathcal{P}_{1}$ be the $m$-set of primes ensured by Proposition 3.3, with elements $p_{1}<\ldots<p_{m}$. Setting all $X_{p}, p \notin \mathcal{P}_{1}$, equal to zero we obtain the identity

$$
Q\left(S_{1}^{*}, \ldots, S_{m}^{*}\right)=P\left(S_{1}^{*}, \ldots, S_{m}^{*}\right) \cdot T^{*}
$$

where $S_{i}^{*}=\psi\left(S\left(\nu, k_{i}\right)\right)\left(X_{p}=0: p \notin \mathcal{P}_{1}\right), i=1, \ldots, m$, and $T^{*}$ are polynomials from $\mathbb{Q}\left[X_{p}: p \in \mathcal{P}_{1}\right]$. Substituting for $X_{p}, p \in \mathcal{P}_{1}$, the polynomial 
$Q_{p}\left(Y_{p}: p \in \mathcal{P}_{1}\right)$ ensured by (a) of Proposition 3.1 and Proposition 3.3, we obtain the identity

$$
Q\left(Y_{p_{1}}, \ldots, Y_{p_{m}}\right)=P\left(Y_{p_{1}}, \ldots, Y_{p_{m}}\right) \cdot T^{* *} .
$$

Hence $P$ divides $Q$.

Lemma 3.4. Let $K$ be an integral domain of characteristic $0, g, d \in \mathbb{N}$, and let $P \in K[X]$ satisfy

$$
P^{g} \in K\left[X^{d}\right] .
$$

Then $P=X^{s} Q\left(X^{d}\right)$ for some integer $s \geq 0$ and a polynomial $Q \in K[X]$.

Proof. We write $P=X^{s} Q$ where $c=Q(0) \neq 0$. From $P^{g} \in K\left[X^{d}\right]$ it follows that $d$ divides $g s$ and $Q^{g} \in K\left[X^{d}\right]$. Suppose, for a contradiction, that $Q \notin K\left[X^{d}\right]$. Let $m>0$ be the smallest integer not divisible by $d$ for which $X^{m}$ has in $Q$ a nonzero coefficient $f$. It is easy to see that the coefficient of $X^{m}$ in $Q^{g}$ is $g c^{g-1} f \neq 0$. This contradicts the assumption $Q^{g} \in K\left[X^{d}\right]$.

Proof of Theorem 1.6. We can assume that $P$ depends on all variables $Y_{1}, \ldots, Y_{m}$ and that if $Q^{d}$ divides $P$ for some $Q \in \mathbb{Q}\left[Y_{1}, \ldots, Y_{m}\right]$ then $Q$ is constant. We set $t=\operatorname{deg}(P)+1$ and $C$ to be $C(m, \operatorname{deg}(P)$ $+1)$ of Proposition 3.3. Suppose that $C<k_{1}<\ldots<k_{m}$ are fixed and $P\left(S\left(n, k_{1}\right), \ldots, S\left(n, k_{m}\right)\right)$ is a $d$ th power of a positive integer for infinitely many $n \in \mathbb{N}$. By Proposition 2.2, there is an integer $e, 0 \leq e<d$, and an element $\tau$ of $\mathcal{E}$ so that

$$
P\left(S\left(e+d \nu, k_{1}\right), \ldots, S\left(e+d \nu, k_{m}\right)\right)=\tau^{d} .
$$

Thus

$$
P\left(S_{1}, \ldots, S_{m}\right)=T^{d}
$$

where $T=\psi(\tau)$ and $S_{i}=\psi\left(S\left(e+d \nu, k_{i}\right)\right)$ are polynomials in $\mathbb{Q}\left[X_{p}: p \in \mathcal{P}\right]$. Let $\mathcal{M}=\left\{I_{1}, \ldots, I_{s}\right\}$ be the interval partition of $[m]$ and $\mathcal{P}_{1}, \ldots, \mathcal{P}_{t}$ be the $t$ disjoint $m$-sets of primes guaranteed by Proposition 3.3. Let $\left\{\alpha_{p} \in \mathbb{C}\right.$ : $p \in \mathcal{P}\}$ be any fixed specialization. We take any of the sets, say $\mathcal{P}_{1}$ with the elements $p_{1}<\ldots<p_{m}$, and set every $X_{p}$ for $p \notin \mathcal{P}_{1}$ equal to $\alpha_{p}$. We get the identity

$$
P\left(S_{1}^{*}, \ldots, S_{m}^{*}\right)=\left(T^{*}\right)^{d}
$$

where $T^{*}$ and $S_{i}^{*}=S_{i}\left(X_{p}:=\alpha_{p}: p \notin \mathcal{P}_{1}\right)$ are polynomials from $\mathbb{Q}\left[X_{p}\right.$ : $\left.p \in \mathcal{P}_{1}\right]$.

Due to the substitution $\nu \mapsto e+d \nu$, actually the polynomial $P\left(S_{1}^{*}, \ldots, S_{m}^{*}\right)$ lies in $\mathbb{Q}\left[X_{p}^{d}: p \in \mathcal{P}_{1}\right]$. Applying the previous lemma (with $g=d$ ) for each of the $m$ variables, we see that

$$
T^{*}=M \cdot U\left(X_{p}^{d}: p \in \mathcal{P}_{1}\right)
$$


where $M=\prod_{p \in \mathcal{P}_{1}} X_{p}^{s_{p}}, s_{p} \geq 0$. From (18) and (19) we obtain, by replacing $X_{p}^{d}$ with the variable $Z_{p}$, that

$$
P\left(U_{1}, \ldots, U_{m}\right)=\prod_{p \in \mathcal{P}_{1}} Z_{p}^{s_{p}} \cdot U^{d}
$$

where $U_{i}, U \in \mathbb{Q}\left[Z_{p}: p \in \mathcal{P}_{1}\right]$. It follows that $\left\{U_{i}: i=1, \ldots, m\right\}$ is an $\mathcal{M}$ regular system. Indeed, $\mathcal{S}=\left\{\psi\left(S\left(\nu, k_{i}\right)\right)\left(X_{p}:=\alpha_{p}: p \notin \mathcal{P}_{1}\right): i=1, \ldots, m\right\}$ is $\mathcal{M}$-regular by Proposition 3.3 and $U_{i}=\psi\left(S\left(e+d \nu, k_{i}\right)\right)\left(X_{p}:=\alpha_{p}: p \notin\right.$ $\left.\mathcal{P}_{1} ; X_{p}^{d}:=Z_{p}: p \in \mathcal{P}_{1}\right)$. That the system $\left\{U_{i}: i=1, \ldots, m\right\}$ satisfies the conditions (i) and (ii) of regularity is clear. Its Jacobian is a nonzero constant because the substitution $\nu \mapsto e+d \nu$ results only in multiplying the column of $X_{p}$ in the diagonal block in the Jacobi matrix of $\mathcal{S}$ by the nonzero constant $p^{e}$. Inverting $\left\{U_{i}: i=1, \ldots, m\right\}$ by (a) of Proposition 3.1, we get the identity

$$
P\left(Z_{p_{1}}, \ldots, Z_{p_{m}}\right)=\prod_{p \in \mathcal{P}_{1}} Q_{p}^{s_{p}} \cdot U\left(Z_{p}:=Q_{p}: p \in \mathcal{P}_{1}\right)^{d}
$$

for some polynomials $Q_{p} \in \mathbb{Q}\left[Z_{p}: p \in \mathcal{P}_{1}\right]$. If $U$ were nonconstant, then $U\left(Z_{p}:=Q_{p}: p \in \mathcal{P}_{1}\right)$ would be nonconstant as well, by (b) and (c) of Proposition 3.1, which is impossible because $P$ is free of all nonconstant $d$ th powers. Hence the polynomial $U$ is a constant different from 0 . Using (18) and (19) we conclude that

$$
P\left(S_{1}^{*}, \ldots, S_{m}^{*}\right)=c \prod_{p \in \mathcal{P}_{1}} X_{p}^{d s_{p}}
$$

where $c \in \mathbb{C}$ is nonzero and $s_{p}$ are nonnegative integers. Not all $s_{p}$ may be zero. Even more is true: By (d) of Proposition 3.1, there exists a $k \in I_{s}$ so that $s_{p_{k}}>0$. Returning to all the variables before the specialization, we conclude that

$$
P\left(S_{1}, \ldots, S_{m}\right)=A \cdot \prod_{p \in \mathcal{P}_{1}} X_{p}^{d s_{p}}+B
$$

where $s_{p_{k}}>0, A, B \in \mathbb{Q}\left[X_{p}: p \in \mathcal{P} \backslash \mathcal{P}_{1}\right]$, and

$$
B\left(X_{p}:=\alpha_{p}: p \in \mathcal{P} \backslash \mathcal{P}_{1}\right)=0 .
$$

Since the expression of $P\left(S_{1}, \ldots, S_{m}\right)$ in the form (20) is unique, the polynomials $A$ and $B$ are independent of the choice of the specialization $\left\{\alpha_{p} \in\right.$ $\mathbb{C}: p \in \mathcal{P}\}$. Since $(21)$ holds for every specialization, $B$ must be a zero polynomial.

Thus there is a $k \in I_{s}$ such that the polynomial $P\left(S_{1}, \ldots, S_{m}\right)$ is divisible by $X_{p_{k}}^{d}$. Since $I_{s}$ is the last part of $\mathcal{M}$, by the remark after Proposition 3.1 the variable $X_{p_{k}}$ appears in every $S_{i}$ only in the term $b X_{p_{k}}^{d}$. Taking any of the sets of primes $\mathcal{P}_{i}, i=1, \ldots, t$, in place of $\mathcal{P}_{1}$, we obtain a set $\mathcal{Q}$ of $t$ primes 
such that (i) the monomial $\left(\prod_{p \in \mathcal{Q}} X_{p}\right)^{d}$ divides $P\left(S_{1}, \ldots, S_{m}\right)$ and (ii) every $X_{p}, p \in \mathcal{Q}$, appears in every $S_{i}$ only in the term $b X_{p}^{d}$. By (i), the degree of $P\left(S_{1}, \ldots, S_{m}\right)$ in the variables $\left\{X_{p}: p \in \mathcal{Q}\right\}$ is at least $d t=d(\operatorname{deg}(P)+1)$. By (ii), this degree is at most $d \operatorname{deg}(P)$. We have a contradiction.

Proof of Theorem 1.7. In view of Theorem 1.6 it suffices to show that if $C_{1}<k_{1}<\ldots<k_{m}$ are distinct then the equality $P\left(S\left(n, k_{1}\right), \ldots, S\left(n, k_{m}\right)\right)$ $=x^{d}$ for $n, x \in \mathbb{N}, x>1$, implies that $d$ is bounded by a constant. We assume that $P$ depends on all $m$ variables and set $C_{1}=C_{1}(P)$ to be equal to the constant $C(m, \operatorname{deg}(P))$ of Theorem 1.6. Suppose that $Q=$ $P\left(\psi\left(S\left(\nu, k_{1}\right)\right), \ldots, \psi\left(S\left(\nu, k_{m}\right)\right)\right)$ is a monomial. We know, by the previous proof, that $Q$ depends on $t=\operatorname{deg}(P)+1$ variables $X_{p}$ such that each of them appears in every $\psi\left(S\left(\nu, k_{i}\right)\right)$ only in the linear term. But then we have the same contradiction for the degree of $Q$ in these variables as before. So $Q$ is not a monomial and $P\left(S\left(\nu, k_{1}\right), \ldots, S\left(\nu, k_{m}\right)\right) \in \mathcal{E}$ has rank at least two. Thus $\left(P\left(S\left(n, k_{1}\right), \ldots, S\left(n, k_{m}\right)\right)\right)_{n \geq 1}$ is a nondegenerate linearly recurrent sequence having a leading root. By a result of Shorey and Stewart [18], it follows that there exists a constant $C_{2}$ which is effectively computable and depends on $P$ and $k_{1}, \ldots, k_{m}$, such that the equality $P\left(S\left(n, k_{1}\right), \ldots, S\left(n, k_{m}\right)\right)=x^{d}, n, x \in \mathbb{N}, x>1$, implies $d<C_{2}$.

\section{References}

[1] M. Aigner and G. Ziegler, Proofs from the Book, Springer, Berlin, 2001.

[2] S. Berg, On snowball sampling, random mappings and related problems, J. Appl. Probab. 18 (1981), 283-290.

[3] D. Branson, Stirling numbers and Bell numbers: Their role in combinatorics and probability, Math. Sci. 25 (2000), 1-31.

[4] B. Brindza and Á. Pintér, On the power values of Stirling numbers, Acta Arith. 60 (1991), 169-175.

[5] E. R. Canfield and C. Pomerance, On the problem of uniqueness for the maximum Stirling number(s) of the second kind, Integers 2 (2002), A1.

[6] P. Corvaja and U. Zannier, Diophantine equations with power sums and universal Hilbert sets, Indag. Math. (N.S.) 9 (1998), 317-332.

[7] P. Erdős, Beweis eines Satzes von Tschebyschef, Acta Sci. Math. (Szeged) 5 (1932), 194-198.

[8] A. van den Essen, To believe or not to believe: The Jacobian conjecture, Rend. Sem. Mat. Torino 55 (1997), 283-290.

[9] H. Iwaniec and J. Pintz, Primes in short intervals, Monatsh. Math. 98 (1984), 115-143.

[10] M. Klazar, Counting pattern-free set partitions. I: A generalization of Stirling numbers of the second kind, European J. Combin. 21 (2000), 367-378.

[11] J. W. Layman and C. L. Prather, Generalized Bell numbers and zeros of successive derivatives of an entire function, J. Math. Anal. Appl. 96 (1983), 42-51.

[12] F. Luca and P. G. Walsh, The product of like-indexed terms in binary recurrences, J. Number Theory 96 (2002), 152-173. 
[13] Á. Pintér, On some arithmetical properties of Stirling numbers, Publ. Math. Debrecen 40 (1992), 91-95.

[14] - On a diophantine problem concerning Stirling numbers, Acta Math. Hungar. 65 (1994), 361-364.

[15] A. J. van der Poorten, Some facts that should be better known, especially about rational functions, in: Number Theory and Applications, R. A. Mollin (ed.), Kluwer, Dordrecht, 1989, 497-528.

[16] J. F. Ritt, A factorization theory for functions $\sum_{i=1}^{n} a_{i} e^{\alpha_{i} z}$, Trans. Amer. Math. Soc. 29 (1927), 584-596.

[17] R. Sánchez-Peregrino, The Lucas congruence for Stirling numbers of the second kind, Acta Arith. 94 (2000), 41-52.

[18] T. N. Shorey and C. L. Stewart, Pure powers in recurrence sequences and some related diophantine equations, J. Number Theory 27 (1987), 324-352.

[19] R. P. Stanley, Enumerative Combinatorics, Vol. 1, Wadsworth \& Brooks/Cole, Monterey, CA, 1986.

[20] L. A. Székely, The analytic behavior of the holiday numbers, Acta Sci. Math. (Szeged) 51 (1987), 365-369.

[21] P. T. Young, Congruences for Bernoulli, Euler, and Stirling numbers, J. Number Theory 78 (1999), 204-227.

Department of Applied Mathematics (KAM) and

Institute for Theoretical Computer Science (ITI)

Charles University

Malostranské náměstí 25

11800 Praha, Czech Republic

E-mail: klazar@kam.mff.cuni.cz
Mathematical Institute, UNAM Ap. Postal 61-3 (Xangari) CP 58089

Morelia, Michoacán, Mexico

E-mail: fluca@matmor.unam.mx

Received on 8.2.2002

and in revised form on 6.5.2002 